\title{
Thrombolytics for venous thromboembolic events: a systematic review with meta-analysis
}

\begin{abstract}
Ariel Izcovich, ${ }^{1}$ Juan M. Criniti, ${ }^{1}$ Federico Popoff, ${ }^{1}$ Liming Lu, ${ }^{2}$ Jiaming Wu, ${ }^{3}$ Walter Ageno, ${ }^{4}$ Daniel M. Witt, ${ }^{5}$ Michael R. Jaff, ${ }^{6}$ Sam Schulman, ${ }^{7-9}$ Veena Manja, ${ }^{10,11}$ Peter Verhamme, ${ }^{12}$ Gabriel Rada, ${ }^{13,14}$ Yuqing Zhang, ${ }^{7}$ Robby Nieuwlaat, ${ }^{7}$ Wojtek Wiercioch, ${ }^{7}$ Holger J. Schünemann, ${ }^{7,15}$ and Ignacio Neumann ${ }^{14}$

${ }^{1}$ Internal Medicine Service, German Hospital, Buenos Aires, Argentina; ${ }^{2}$ Clinical Research and Data Center, South China Research Center for Acupuncture and Moxibustion,

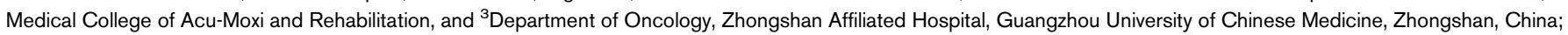
${ }^{4}$ Department of Medicine and Surgery, University of Insubria, Varese, Italy; ${ }^{5}$ Department of Pharmacotherapy, University of Utah College of Pharmacy, Salt Lake City, UT;

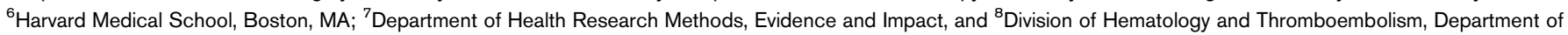

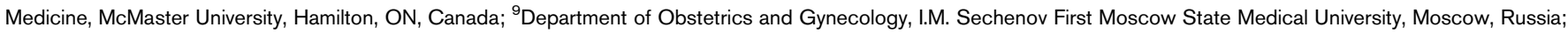
${ }^{10}$ Department of Surgery, University of California Davis, Sacramento, CA; ${ }^{11}$ Department of Medicine, Veterans Affairs Northern California Health Care System, Mather, CA;

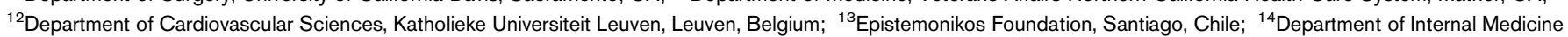
and Centro Evidencia Unversidad Católica, Pontificia Universidad Católica de Chile, Santiago, Chile; and ${ }^{15}$ Department of Medicine, McMaster University, Hamilton, ON, Canada
\end{abstract}

\section{Key Points}

- In patients with submassive- or intermediate-risk PE, thrombolytics probably reduce mortality.

- In patients with VTEs (PE or DVT), thrombolytics increase the risk of major bleeding, including intracranial bleeding.
Thrombolytic therapy might reduce venous thromboembolism-related mortality and morbidity, but it could also increase the risk of major bleeding. We systematically reviewed the literature to evaluate the effectiveness and safety of thrombolytics in patients with pulmonary embolism (PE) and/or deep venous thrombosis (DVT). We searched Medline, Embase, and Cochrane databases for relevant randomized controlled trials up to February 2019. Multiple investigators independently screened and collected data. We included 45 studies (4740 participants). Pooled estimates of PE studies indicate probable reduction in mortality with thrombolysis (risk ratio [RR], 0.61; 95\% confidence interval [CI], 0.40-0.94) (moderate certainty) and possible reduction in nonfatal PE recurrence (RR, 0.56; 95\% CI, 0.35-0.89) (low certainty). Pooled estimates of DVT studies indicate the possible absence of effects on mortality (RR, 0.77; 95\% CI, 0.26-2.28) (low certainty) and recurrent DVT (RR, 0.99; 95\% CI, 0.56-1.76) (low certainty), but possible reduction in postthrombotic syndrome (PTS) with thrombolytics (RR, 0.70 ; 95\% CI, 0.59-0.83) (low certainty). Pooled estimates of the complete body of evidence indicate increases in major bleeding (RR, 1.89; 95\% CI, 1.46-2.46) (high certainty) and a probable increase in intracranial bleeding (RR, 3.17; 95\% CI 1.19-8.41) (moderate certainty) with thrombolytics. Our findings indicate that thrombolytics probably reduce mortality in patients with submassive- or intermediate-risk PE and may reduce PTS in patients with proximal DVT at the expense of a significant increase in major bleeding. Because the balance between benefits and harms is profoundly influenced by the baseline risks of critical outcomes, stakeholders involved in decision making would need to weigh these effects to define which clinical scenarios merit the use of thrombolytics.

\section{Introduction}

Deep venous thrombosis (DVT) and pulmonary embolism (PE) are major contributors to global disease burden. ${ }^{1}$ Although PE can be life-threatening, both conditions are accompanied by significant morbidity
Submitted 17 January 2020; accepted 24 February 2020; published online 14 April 2020. DOI 10.1182/bloodadvances.2020001513.
For original data, please contact the corresponding author, Ariel Izcovich, by e-mail at ariel.izcovich@gmail.com.

The full-text version of this article contains a data supplement. 
(dyspnea, chronic thromboembolic pulmonary hypertension, edema, and postthrombotic syndrome [PTS]). Standard treatment with anticoagulation has the main objective of preventing a recurrent venous thromboembolism (VTE). Despite treatment, approximately $10 \%$ of patients with an unprovoked event will present with a recurrent VTE during the first year, ${ }^{2}$ and a significant number of patients with DVT may suffer postthrombotic symptoms in the long term, manifested by some degree of pain, swelling, skin pigmentation, or venous ulceration of the affected limb. ${ }^{3,4}$ Thrombolytic agents dissolve blood clots by activating plasminogen. Clot dissolution may restore pulmonary arterial flow, which improves right ventricular hemodynamics in patients with PE and reduces the risk of permanent damage to the vein (and venous valve) structure and function in patients with DVT. Both of these effects may have a positive impact on important patient outcomes such as mortality, PTS, and risk of VTE recurrence. However, thrombolytics may increase the risk of major bleeding. Therefore, the potential impact of thrombolytic drugs on therapeutic decision making during VTE treatment requires a detailed analysis of the body of existing evidence. In the context of the development of the recommendations on treatment of VTEs for the American Society of Hematology (ASH), we examined the evidence regarding the effect of thrombolytic therapy on important outcomes in patients with PE and/or DVT.

\section{Methods}

This systematic review was performed as part of the ASH Guidelines on VTE, which were developed in partnership with McMaster University's Grading of Recommendations Assessment, Development and Evaluation (GRADE) Centre. Review and metaanalysis methodology followed the Cochrane Handbook, ${ }^{5}$ with reporting according to the Preferred Reporting Items for Systematic Reviews and Meta-Analyses (PRISMA) guidelines. ${ }^{6}$

\section{Data sources and searches}

We updated searches from 2 previous systematic reviews that included articles up to December 2018 (PE studies) ${ }^{7}$ and February 2016 (DVT studies). ${ }^{8}$ We complemented those reviews with a search of Medline, Medline in-process, Embase, and Cochrane Central Register of Controlled Trials (CENTRAL) from 1 March 2016 to 12 February 2019, using a combination of keywords for "venous thromboembolic events" AND "thrombolytic therapy". Searches were not limited by language or publication type. We also searched all references from included studies and studies citing the included studies on Google Scholar. In addition, we conducted a search in the Epistemonikos database (see supplemental Data for the detailed search strategy) and asked the panelists from the ASH Guideline Panel for additional trials not identified in the electronic search.

\section{Study selection and inclusion criteria}

Two reviewers (A.I. and J.M.C.) addressed eligibility and data abstraction using a standardized abstraction form. We included randomized controlled trials that compared treatment with any thrombolytic agent with placebo or no thrombolytic therapy in patients of any age with a diagnosis of PE or DVT.

\section{Outcomes measured}

We included studies that evaluated at least 1 of the following patient-important outcomes in both thrombolytic and comparator arms. For patients with PE, we included studies on overall mortality, recurrent nonfatal PE, DVT, functional class limitation (New York Heart Association [NYHA] III-IV), major bleeding, and intracranial bleeding as a subgroup of major bleeding. For patients with DVT, we included studies on short-term mortality, defined as any death occurring in the first 30 days of treatment; late mortality, defined as all-cause mortality excluding events occurring in the first 30 days; nonfatal PE; recurrent DVT; PTS; leg ulceration; major bleeding; and intracranial bleeding as a subgroup of major bleeding. For PE and DVT outcomes, we considered both symptomatic and asymptomatic events.

\section{Data extraction, quality assessment, and certainty of evidence}

Five reviewers (A.I., J.M.C., F.P., L.L., and J.W.) independently and in duplicate extracted trial details pertaining to the participants, interventions, comparators, and results, and they assessed the certainty in estimates of effect. We followed the GRADE Working Group guidance and judged the certainty in the estimates of effect considering issues of risk of bias, precision, consistency, directness, and publication bias for each individual outcome.

Assessment of risk of bias. Risk of bias was assessed independently by 2 reviewers on an outcome basis by using a modification of the Cochrane Risk of Bias Tool 1.0, which considers the following 5 questions to determine whether there was high, moderate, or low risk of bias in the included studies: (1) Was the allocation sequence adequately generated? (2) Was allocation adequately concealed? (3) Blinding: Was knowledge of the allocated interventions adequately prevented? (4) Was loss to follow-up (missing outcome data) infrequent? How was the missing data handled? (see "Handling of missing information"); and (5) Were other potential sources of bias identified? Except for missing information, response options were Definitely yes (low risk of bias), Probably yes (low risk of bias), Probably no (high risk of bias), and Definitely no (high risk of bias). ${ }^{9}$

Handling of missing information. To assess the impact of missing outcome data in the included studies, we planned to perform a sensitivity analysis for each of the efficacy outcomes to evaluate the robustness of the results as suggested by the GRADE Working Group. ${ }^{10}$ Whenever missing data imputation strategies did not significantly affect the observed effect, we answered the assessment of risk of bias for missing information as "Definitely yes" (low risk of bias) for all the pooled trials. When a trial did not provide the necessary information to perform the mentioned sensitivity analysis, we excluded the missing patient information and answered the assessment of risk of bias for missing information as "Definitely no" (high risk of bias) for that trial.

Precision. We rated down the confidence in estimate of effects for imprecision if the 95\% confidence interval $(\mathrm{Cl})$ included both appreciable benefits and harms and/or if the body of evidence did not meet the optimal information size (OIS) for a specific outcome. We calculated OIS using $\alpha(0.05)$ and $\beta(0.20)$ values and a relative risk difference of $25 \%$ with the median of the control event rate for dichotomous outcomes. ${ }^{11}$

\section{Assessment of reporting biases}

We assessed the presence of publication bias considering the size and sponsorship of the included studies and the identification of 
Figure 1. Study flow diagram. SR, systematic review.

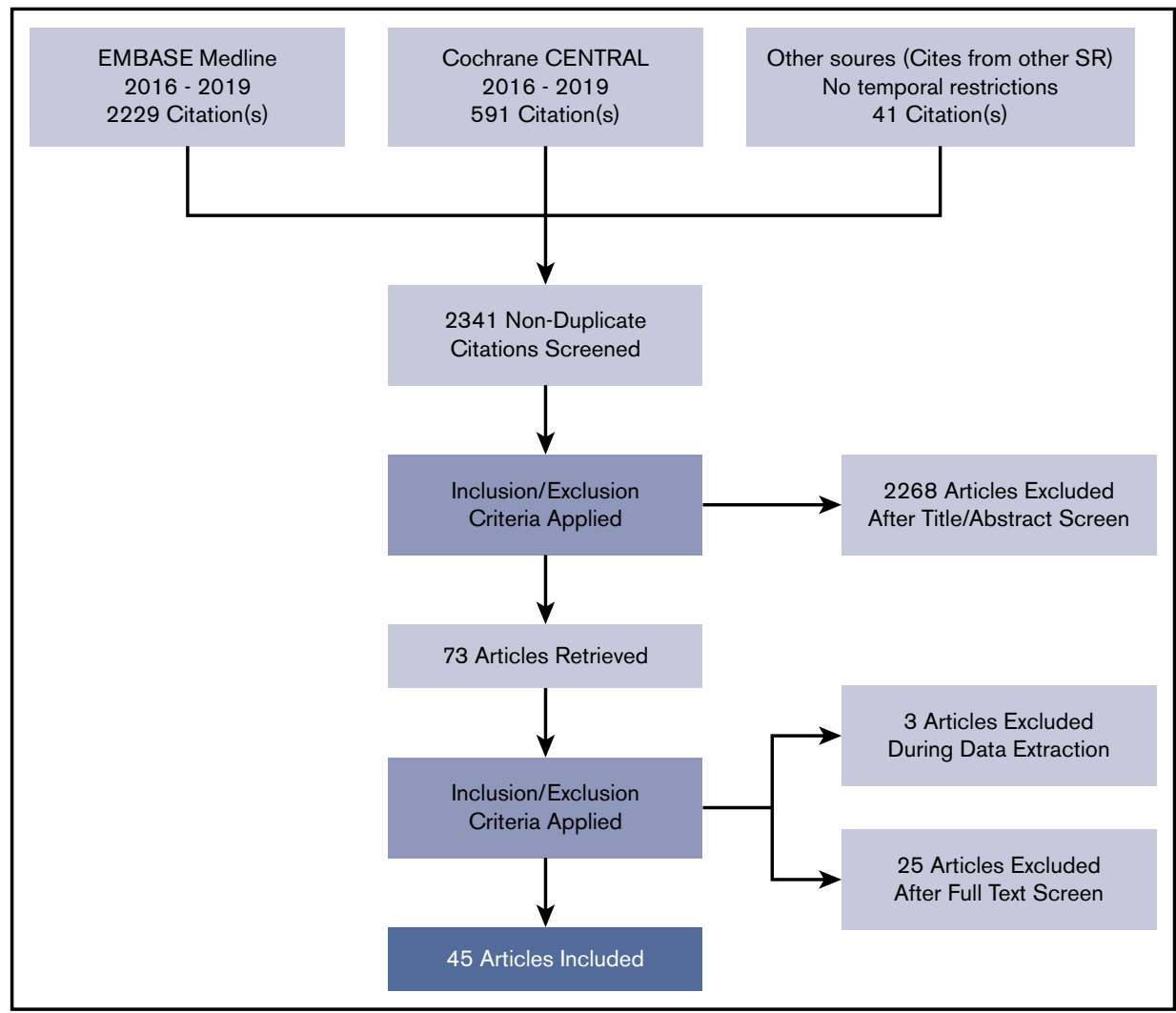

unpublished studies for which sponsors withheld results. ${ }^{12}$ When 6 or more eligible trials were available, we constructed funnel plots. We also considered the presence of selective outcome reporting when critically important outcomes were not reported in most of the trials or were reported in a way that could not be pooled.

\section{Handling of discrepancies and data synthesis and analysis}

We resolved discrepancies in eligibility, data abstraction, risk of bias, and GRADE certainty of evidence assessments by discussion. We conducted a complete case analysis and performed sensitivity analyses with different imputation strategies in cases of missing data as already described. All pooled estimates used random effects models provided by ReviewManager (RevMan version 5.3; The Nordic Cochrane Centre, The Cochrane Collaboration, 2012, Copenhagen, Denmark). The statistical method used was MantelHaenszel. For calculating risk differences (RDs), we applied relative effects-the mean control group risk estimate from the included studies. $^{13}$

\section{Heterogeneity}

To quantify the inconsistency among the pooled estimates, we used the $\chi^{2}$ test and the $I^{2}$ statistic. We conducted subgroup analysis on the basis of the following four a priori hypotheses when five or more trials were pooled: (1) risk of bias: we anticipated smaller effects for efficacy outcomes and larger effects for safety outcomes in publications with low risk of bias; (2) thrombolytic type: we anticipated bigger effects and fewer adverse effects with more fibrin-specific plasminogen activators (recombinant tissue plasminogen activator [rt-PA] and tenecteplase) as opposed to streptokinase and urokinase; (3) VTE event severity: we anticipated larger effects in patients with more severe events (hemodynamic instability, submassive- or intermediate-risk PE, iliofemoral DVT); and (4) type of thrombolytic infusion: we anticipated larger effect and fewer adverse effects with catheterdirected infusion. For safety outcomes we also conducted a subgroup analysis comparing trials that included patients with PE and DVT. For each subgroup analysis, we tested for interaction by using a $\chi^{2}$ significance test.

\section{Results}

After excluding the duplicate and irrelevant publications by title, we identified and assessed 2341 references for inclusion. Of these, we excluded 2296 (Figure 1) leaving $45^{14-58}$ trials for data extraction, 26 trials $^{14-39}$ included patients with PE, and 19 trials ${ }^{40-58}$ included patients with DVT (Tables 1 and 2).

\section{PE trials}

Study characteristics. The 26 included trials were published between 1971 and 2018 and recruited 2784 patients (median of 58 patients per study) who had a mean age of 45 to 68 years, and the proportion of females ranged from $25.5 \%$ to $66.6 \%$ (Table 1). One small study included only patients with massive PE (hemodynamic instability), ${ }^{19}$ 16 studies included patients with submassive- or intermediate-risk PE (right ventricular dysfunction [RVD] and/or myocardial injury without hemodynamic compromise), ${ }^{14,17,20-22,24,25,27,29-31,34-36,38,39} 1$ study included patients with or without submassive- or intermediaterisk $\mathrm{PE}$ and reported on the subgroup of hemodynamically stable 


\begin{tabular}{|c|c|c|c|c|c|c|}
\hline References & $\begin{array}{l}\text { Treatment (no. of } \\
\text { participants) }\end{array}$ & $\begin{array}{l}\text { Mean } \\
\text { age, y }\end{array}$ & $\begin{array}{l}\text { Females in } \\
\text { study, } \%\end{array}$ & PE characteristics & Thrombolytic scheme & Infusion type \\
\hline Becattini et al ${ }^{14}$ & $\begin{array}{l}\text { Thrombolytic plus UFH }(n=28) \\
\text { placebo plus UFH }(n=30)\end{array}$ & 68 & 39.1 & $\begin{array}{l}\text { Acute submassive- or intermediate- } \\
\text { risk PE defined as RVD } \\
\text { (echocardiography) without } \\
\text { systolic hypotension }\end{array}$ & Tenecteplase $30-50$ mg (bolus) & Systemic \\
\hline 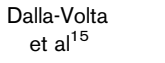 & $\begin{array}{l}\text { Thrombolytic plus UFH }(n=20) \text {; } \\
\text { UFH alone }(n=16)\end{array}$ & 65 & 66.6 & $\begin{array}{l}\text { Acute PE (any) without systolic } \\
\text { hypotension }\end{array}$ & $\begin{array}{l}\text { rt-PA } 100 \mathrm{mg} \text { (bolus }+2-\mathrm{h} \\
\text { infusion) }\end{array}$ & Systemic \\
\hline Dotter et $\mathrm{al}^{16}$ & $\begin{array}{l}\text { Thrombolytic plus UFH }(n=15) \\
\text { UFH alone }(n=16)\end{array}$ & $\begin{array}{c}\text { Range, } \\
18-85\end{array}$ & 61.3 & $\begin{array}{l}\text { Acute PE (any) without systolic } \\
\text { hypotension }\end{array}$ & $\begin{array}{l}\text { Streptokinase } 2550 \text { 000-11 } \\
450000 \text { IU (bolus }+18 \text { - to } \\
72-\mathrm{h} \text { infusion) }\end{array}$ & Systemic \\
\hline Fasullo et al $^{17}$ & $\begin{array}{l}\text { Thrombolytic plus UFH }(n=37) \\
\text { placebo plus UFH }(n=35)\end{array}$ & 56 & 51.3 & $\begin{array}{l}\text { Acute submassive- or intermediate- } \\
\text { risk PE defined as RVD } \\
\text { (echocardiography) without } \\
\text { systolic hypotension }\end{array}$ & $\begin{array}{l}\mathrm{rt}-\mathrm{PA} 100 \mathrm{mg} \text { (bolus }+2-\mathrm{h} \\
\text { infusion) }\end{array}$ & Systemic \\
\hline $\begin{array}{l}\text { Goldhaber } \\
{\text { et } \mathrm{al}^{18}}^{18}\end{array}$ & $\begin{array}{l}\text { Thrombolytic plus UFH }(n=46) \text {; } \\
\text { UFH alone }(n=55)\end{array}$ & 59 & 56.4 & $\begin{array}{l}\text { Acute PE (any) without systolic } \\
\text { hypotension; } 45.5 \% \text { had } \\
\text { submassive- or intermediate-risk } \\
\text { PE defined as RV hypokinesis } \\
\text { (echocardiography) }\end{array}$ & rt-PA $100 \mathrm{mg}$ (2-h infusion) & Systemic \\
\hline $\begin{array}{l}\text { Jerjes-Sanchez } \\
\quad \text { et } \mathrm{al}^{19}\end{array}$ & $\begin{array}{l}\text { Thrombolytic plus UFH }(n=4) \text {; } \\
\text { UFH alone }(n=4)\end{array}$ & 48 & 37.5 & $\begin{array}{l}\text { Acute massive } \mathrm{PE} \text { defined as }>9 \\
\text { obstructed segments on V/Q lung } \\
\text { scan with or without cardiogenic } \\
\text { shock (systolic } \mathrm{BP}<90 \mathrm{mmHg} \text { ) } \\
\text { (all included patients had } \\
\text { cardiogenic shock) }\end{array}$ & $\begin{array}{l}\text { Streptokinase } 1500000 \mathrm{IU} \\
\quad(1-\mathrm{h} \text { infusion) }\end{array}$ & Systemic \\
\hline Kline et $\mathrm{al}^{20}$ & $\begin{array}{l}\text { Thrombolytic plus } \mathrm{LMWH}(\mathrm{n}=40) \\
\text { heparin plus } \mathrm{LMWH}(\mathrm{n}=43)\end{array}$ & 55 & 39.7 & $\begin{array}{l}\text { Acute submassive- or intermediate- } \\
\text { risk PE defined as RVD } \\
\text { (echocardiography) or myocardial } \\
\text { injury (BNP) without systolic } \\
\text { hypotension }\end{array}$ & Tenecteplase $30-50 \mathrm{mg}$ (bolus) & Systemic \\
\hline $\begin{array}{l}\text { Kostantinides } \\
\text { et } \mathrm{al}^{21}\end{array}$ & $\begin{array}{l}\text { Thrombolytic plus UFH }(n=118) \\
\text { placebo plus UFH }(n=138)\end{array}$ & 62 & 52.3 & $\begin{array}{l}\text { Acute submassive- or intermediate- } \\
\text { risk PE defined as RVD } \\
\text { (echocardiography) without } \\
\text { systolic hypotension }\end{array}$ & $\begin{array}{l}\text { rt-PA } 100 \mathrm{mg} \text { (bolus }+2-\mathrm{h} \\
\text { infusion) }\end{array}$ & Systemic \\
\hline Kucher et $\mathrm{al}^{22}$ & $\begin{array}{l}\text { Thrombolytic plus UFH }(n=30) \\
\text { UFH alone }(n=29)\end{array}$ & 63 & 49.1 & $\begin{array}{l}\text { Acute submassive- or intermediate- } \\
\text { risk PE defined as RVD } \\
\text { (echocardiography) without } \\
\text { systolic hypotension }\end{array}$ & rt-PA 10-20 mg (15-h infusion) & $\begin{array}{l}\text { Directed } \\
\text { (ultrasound } \\
\text { assisted) }\end{array}$ \\
\hline Levine et $\mathrm{al}^{23}$ & $\begin{array}{l}\text { Thrombolytic plus UFH }(n=33) \text {; } \\
\text { placebo plus UFH }(n=25)\end{array}$ & 61 & 50.0 & $\begin{array}{l}\text { Acute PE (any) without systolic } \\
\text { hypotension }\end{array}$ & $\begin{array}{l}\mathrm{rt}-\mathrm{PA} 0.6 \mathrm{mg} / \mathrm{kg} \text { ideal body weight } \\
\text { (bolus) }\end{array}$ & Systematic \\
\hline Liu et $\mathrm{a}^{24}$ & $\begin{array}{l}\text { Thrombolytic plus LMWH }(n=30) \\
\text { LMWH alone }(n=24)\end{array}$ & 58.2 & $\begin{array}{l}\text { No significant } \\
\text { differences } \\
\text { between sexes }\end{array}$ & $\begin{array}{l}\text { Acute submassive- or intermediate- } \\
\text { risk PE defined as RVD } \\
\text { (echocardiography) without } \\
\text { systolic hypotension }\end{array}$ & rt-PA 50 mg (bolus) & Systemic \\
\hline Lu et $\mathrm{al}^{25}$ & $\begin{array}{l}\text { Thrombolytic plus LMWH }(n=51) \text {; } \\
\text { LMWH alone }(n=55)\end{array}$ & 60.8 & 25.5 & $\begin{array}{l}\text { Acute submassive- or intermediate- } \\
\text { risk PE defined as RVD } \\
\text { (echocardiography) without } \\
\text { systolic hypotension }\end{array}$ & Urokinase $20000 \mathrm{lU} / \mathrm{kg}$ (bolus) & Systemic \\
\hline Ly et $\mathrm{al}^{26}$ & $\begin{array}{l}\text { Thrombolytic plus UFH }(n=14) \text {; } \\
\text { UFH alone }(n=10)\end{array}$ & 53.2 & 56 & $\begin{array}{l}\text { Acute PE (any) without systolic } \\
\text { hypotension. }\end{array}$ & $\begin{array}{l}\text { Streptokinase } 7450000 \mathrm{IU} \\
\quad \text { (bolus }+72 \text {-h infusion) }\end{array}$ & Systemic \\
\hline Meyer et $\mathrm{al}^{27}$ & $\begin{array}{l}\text { Thrombolytic plus UFH }(n=506) \\
\text { placebo plus UFH }(n=499)\end{array}$ & 66.2 & 52.9 & $\begin{array}{l}\text { Acute submassive- or intermediate- } \\
\text { risk PE defined as RVD } \\
\text { (echocardiography or CT) plus } \\
\text { myocardial injury (troponin) without } \\
\text { systolic hypotension }\end{array}$ & Tenecteplase $30-50 \mathrm{mg}$ (bolus) & Systemic \\
\hline PIOPED $^{28}$ & $\begin{array}{r}\text { Thrombolytic plus UFH }(n=9) \\
\text { placebo plus heparin }(n=4)\end{array}$ & 58.5 & 30.8 & $\begin{array}{l}\text { Acute PE (any) without systolic } \\
\text { hypotension. }\end{array}$ & $\begin{array}{l}\text { rt-PA } 40-80 \mathrm{mg} \text { (40- to } 90-\mathrm{min} \\
\text { infusion) }\end{array}$ & Systemic \\
\hline Sharifi et $\mathrm{al}^{29}$ & $\begin{array}{l}\text { Thrombolytic plus UFH }(n=61) \\
\text { UFH alone }(n=60)\end{array}$ & 58.5 & 54.5 & $\begin{array}{l}\text { Acute submassive- or intermediate- } \\
\text { risk PE clinically defined (chest } \\
\text { pain, tachypnea, tachycardia, } \\
\text { dyspnea, cough oxygen } \\
\text { desaturation or elevated venous } \\
\text { pressure) or RVD } \\
\text { (echocardiography) or myocardial } \\
\text { injury (troponin or BNP) without } \\
\text { systolic hypotension }\end{array}$ & $\mathrm{rt}-\mathrm{PA} 50 \mathrm{mg}$ (bolus + 2-h infusion) & Systemic \\
\hline
\end{tabular}

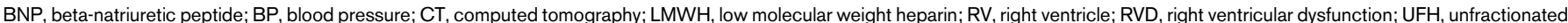
heparin; VQ, ventilation/perfusion.

*Study did not report mean age. 


\begin{tabular}{|c|c|c|c|c|c|c|}
\hline References & $\begin{array}{l}\text { Treatment (no. of } \\
\text { participants) }\end{array}$ & $\begin{array}{l}\text { Mean } \\
\text { age, } y\end{array}$ & $\begin{array}{l}\text { Females in } \\
\text { study, } \%\end{array}$ & PE characteristics & Thrombolytic scheme & Infusion type \\
\hline $\operatorname{Sun}^{30}$ & $\begin{array}{l}\text { Thrombolytic plus LMWH }(n=46) \text {; } \\
\text { LMWH alone }(n=46)\end{array}$ & 56.3 & 44.6 & $\begin{array}{l}\text { Acute submassive- or intermediate- } \\
\text { risk PE defined as RVD } \\
\text { (echocardiography) without } \\
\text { systolic hypotension }\end{array}$ & Urokinase 500000 IU (bolus) & Systemic \\
\hline $\begin{array}{l}\text { Taherkhani } \\
\text { et } \mathrm{al}^{31}\end{array}$ & $\begin{array}{l}\text { Thrombolytic plus UFH }(n=25) \text {; } \\
\text { UFH alone }(n=25)\end{array}$ & 55.7 & 60 & $\begin{array}{l}\text { Acute submassive- or intermediate- } \\
\text { risk PE defined as RVD or } \\
\text { enlargement (echocardiography) } \\
\text { without systolic hypotension }\end{array}$ & $\begin{array}{l}\text { rt-PA } 100 \mathrm{mg} \text { (90-min infusion) or } \\
\text { streptokinase } 1500000(2-\mathrm{h} \\
\text { infusion) }\end{array}$ & Systemic \\
\hline Tibutt et $\mathrm{al}^{32}$ & $\begin{array}{l}\text { Thrombolytic }(n=13) ; \text { UFH } \\
\qquad(n=17)\end{array}$ & 48.7 & 50 & $\begin{array}{l}\text { Acute PE (any); mean systolic BP } \\
108 \mathrm{mmHg}\end{array}$ & $\begin{array}{l}\text { Streptokinase } 600000 \mathrm{IU} \text { (bolus } \\
\quad+72 \text {-h infusion) }\end{array}$ & $\begin{array}{l}\text { Infused in the } \\
\text { pulmonary artery }\end{array}$ \\
\hline UKEP ${ }^{33}$ & $\begin{array}{l}\text { Thrombolytic }(n=82) ; \text { UFH } \\
\quad(n=78)\end{array}$ & $\begin{array}{l}49.4 \% \\
>50 y^{*}\end{array}$ & 42.5 & $\begin{array}{l}\text { Acute PE (any); } 8.7 \% \text { with } \\
\text { hemodynamic compromise }\end{array}$ & $\begin{array}{l}\text { Urokinase } 2000 \text { (12-h infusion); } \\
\quad 4400 \mathrm{IU} / \mathrm{kg} \text { (24-h infusion) }\end{array}$ & $\begin{array}{l}\text { Infused in the } \\
\text { pulmonary artery }\end{array}$ \\
\hline Wei and $\operatorname{Sun}^{34}$ & $\begin{array}{l}\text { Thrombolytic ( } n=28) ; \text { LMWH } \\
\quad(n=28) ; 2 \text { lost to follow-up in the } \\
\text { control group }\end{array}$ & 58 & 40.0 & $\begin{array}{l}\text { Acute submassive- or intermediate- } \\
\text { risk PE defined as RVD } \\
\text { (echocardiography) without } \\
\text { systolic hypotension }\end{array}$ & rt-PA 50 mg (bolus) & Systemic \\
\hline Zhang et $\mathrm{al}^{35}$ & $\begin{array}{l}\text { Thrombolytic }(n=26) ; \text { LMWH } \\
\qquad(n=29)\end{array}$ & 68 & 41.8 & $\begin{array}{l}\text { Acute submassive- or intermediate- } \\
\text { risk PE defined as RVD } \\
\text { (echocardiography) without } \\
\text { systolic hypotension }\end{array}$ & rt-PA 50 mg (bolus) & Systemic \\
\hline $\mathrm{Ou}^{36}$ & $\begin{array}{l}\text { Thrombolytic }(n=54) ; \text { UFH } \\
\quad(n=54)\end{array}$ & 58.3 & 28.7 & $\begin{array}{l}\text { Acute submassive- or intermediate- } \\
\text { risk PE defined as RVD } \\
\text { (echocardiography) without } \\
\text { systolic hypotension }\end{array}$ & Urokinase $20000 \mathrm{IU} / \mathrm{kg}$ (bolus) & Systemic \\
\hline Marini et $\mathrm{al}^{37}$ & $\begin{array}{l}\text { Thrombolytic }(n=20) ; \text { UFH } \\
\quad(n=10)\end{array}$ & 53 & 40.0 & $\begin{array}{l}\text { Acute PE (any) without systolic } \\
\text { hypotension. }\end{array}$ & $\begin{array}{l}\text { Urokinase } 2400000 \mathrm{IU} \\
\quad \text { (36-h infusion) }\end{array}$ & Systemic \\
\hline Ahmed et $\mathrm{al}^{38}$ & $\begin{array}{l}\text { Thrombolytic }(n=24) ; \text { UFH } \\
\quad(n=28)\end{array}$ & 44.7 & 53.8 & $\begin{array}{l}\text { Acute submassive- or intermediate- } \\
\text { risk PE defined as RVD } \\
\text { (echocardiography) or myocardial } \\
\text { injury (troponin or BNP) without } \\
\text { systolic hypotension }\end{array}$ & $\begin{array}{l}\text { Streptokinase } 1500000 \mathrm{IU} \\
\text { (2-h infusion) }\end{array}$ & Systemic \\
\hline Zhang et $\mathrm{al}^{39}$ & $\begin{array}{l}\text { Thrombolytic }(n=33) ; \text { LMWH } \\
\qquad(n=33)\end{array}$ & 59.5 & 51.5 & $\begin{array}{l}\text { Acute intermediate-risk PE defined } \\
\text { as RVD (echocardiography) or } \\
\text { myocardial injury (troponin or BNP) } \\
\text { without systolic hypotension }\end{array}$ & rt-PA 30 mg (2-h infusion) & Systemic \\
\hline
\end{tabular}

BNP, beta-natriuretic peptide; BP, blood pressure; CT, computed tomography; LMWH, low molecular weight heparin; RV, right ventricle; RVD, right ventricular dysfunction; UFH, unfractionated heparin; VQ, ventilation/perfusion.

*Study did not report mean age.

patients with no criteria for submassive or intermediate risk, ${ }^{18}$ and 9 studies $^{15,16,18,23,26,28,32,33,37}$ did not specify PE event characteristics.

Risk of bias. Of the 26 studies included, we classified $5^{14,17,20,21,27}$ as having a low risk of bias (supplemental Figure 1). In the remaining studies, investigators, patients, and outcome assessors were not blinded. We also considered that randomization and treatment allocation concealment was inappropriate in some of these studies (supplemental Figure 1). Sensitivity analyses that considered patients who were lost to follow-up were not performed because only 2 patients met this criterion in 1 of the included studies. Although most of the trials (60\%-75\%) did not blind participants and/or study investigators, we decided not to rate down the certainty of the evidence for mortality, DVT, and limited functional outcomes for 3 reasons: (1) no significant differences between trials with moderate/high and low risk of bias were observed in the subgroup analysis; (2) the most relevant study limitation was lack of blinding, which may have less of an impact on objective outcomes such as mortality; and (3) although studies with a low risk of bias made up less than one fifth of the trials included, they contributed substantially to the pooled estimates $(48.7 \%$ of the mortality outcome meta-analysis weight, $63.9 \%$ of the DVT outcome meta-analysis weight, and $100 \%$ of the limited functional class outcome meta-analysis weight).

\section{DVT trials}

Study characteristics. The 19 included trials were published between 1978 and 2017 and recruited 1956 patients (median of 48 patients per study) who had a mean age of 39.6 to 68.5 years, and the proportion of females ranged from $14.7 \%$ to $69.5 \%$ (Table 2). Twelve trials included patients with proximal DVT, ${ }^{40,42-46,51,52,54,56-58} 1$ trial included only patients with distal DVT, 50 and in 6 trials, thrombus location or extension was not used as an inclusion or exclusion criterion. ${ }^{41,47-49,53,55}$ Although thrombolytics were infused systemically in most studies ( $n=13$ ), catheter-directed infusion was used in 4 studies, and local infusion in the dorsal pedal vein was used in 2 studies.

Risk of bias. Of the 19 included studies, we classified only 2 of them as having low risk of bias (supplemental Figure 2). ${ }^{54,57}$ In the remaining studies, investigators, patients, and outcome assessors were not blinded, or randomization and treatment allocation concealment was judged as inappropriate (supplemental Figure 2). Sensitivity analyses considering patients lost to follow-up were 


\begin{tabular}{|c|c|c|c|c|c|c|}
\hline References & No. of participants & $\begin{array}{l}\text { Mean } \\
\text { age, y }\end{array}$ & $\begin{array}{l}\text { Females in } \\
\text { study, } \%\end{array}$ & DVT extension & Thrombolytic scheme & Infusion type \\
\hline Arnesen et al ${ }^{40}$ & $\begin{array}{l}\text { Thrombolytic plus UFH }(n=21) \\
\text { UFH }(n=21)\end{array}$ & 49.5 & 35.7 & $\begin{array}{l}\text { Proximal DVT, defined as } \\
\text { proximal extension beyond the } \\
\text { calf veins }\end{array}$ & $\begin{array}{l}\text { Streptokinase } 7450000 \mathrm{IU} \text { (bolus } \\
\quad+72 \text {-h infusion) }\end{array}$ & Systemic \\
\hline Common et $\mathrm{al}^{41}$ & $\begin{array}{l}\text { Thrombolytic plus UFH }(n=22) \text {; } \\
\text { UFH }(n=26)\end{array}$ & 50.2 & 33.3 & Any DVT (lower limb) & $\begin{array}{l}\text { Streptokinase; treatment scheme } \\
\text { not specified }\end{array}$ & Systemic \\
\hline Elliot et al ${ }^{42}$ & $\begin{array}{l}\text { Thrombolytic plus UFH }(n=26) \text {; } \\
\text { UFH }(n=25)\end{array}$ & 49.5 & 33.3 & $\begin{array}{l}\text { Proximal DVT, defined as } \\
\text { extension beyond the axial } \\
\text { veins of the calf or forearm }\end{array}$ & $\begin{array}{l}\text { Streptokinase } 7800000 \mathrm{IU} \text { (bolus } \\
\quad+72 \text {-h infusion) }\end{array}$ & Systemic \\
\hline $\begin{array}{l}\text { Elsharawy and } \\
\text { Elzayat }^{43}\end{array}$ & $\begin{array}{l}\text { Thrombolytic plus UFH }(n=18) \\
\text { UFH }(n=17)\end{array}$ & 46.5 & 69 & $\begin{array}{l}\text { Proximal DVT, iliofemoral venous } \\
\text { thrombosis documented with } \\
\text { color duplex and/or ascending } \\
\text { venography }\end{array}$ & $\begin{array}{l}\text { Streptokinase } 2900000 \mathrm{IU} \text { (bolus } \\
\quad+12 \text { - to } 24 \text {-h infusion) }\end{array}$ & Directed \\
\hline Enden et $\mathrm{al}^{44}$ & $\begin{array}{l}\text { Thrombolytic plus LMWH }(n=93) \\
\text { (101 were originally allocated } \\
\text { but only } 93 \text { received the } \\
\text { intervention); LMWH }(n=108)\end{array}$ & 55.5 & 54 & $\begin{array}{l}\text { Proximal DVT, defined as } \\
\text { thrombosis localized in } \\
\text { the upper half of the thigh, } \\
\text { the common iliac vein, or the } \\
\text { combined iliofemoral segment }\end{array}$ & $\begin{array}{l}\text { rt-PA } 20 \text { to } 80 \mathrm{mg} \text { (bolus }+24 \text { - to } \\
\text { 96-h infusion) }\end{array}$ & Directed \\
\hline $\begin{array}{l}\text { Goldhaber } \\
\text { et } \mathrm{al}^{45}\end{array}$ & $\begin{array}{l}\text { Thrombolytic plus UFH }(n=8) \text {; } \\
\text { UFH }(n=9)\end{array}$ & 68.5 & 64.7 & $\begin{array}{l}\text { Proximal DVT, defined as } \\
\text { thrombosis of the popliteal, } \\
\text { femoral, or iliac veins with or } \\
\text { without concomitant calf vein } \\
\text { thrombosis; upper extremity } \\
\text { DVT, defined as thrombosis of } \\
\text { the brachial, axillary, subclavian, } \\
\text { or internal jugular veins }\end{array}$ & $\begin{array}{l}\text { Urokinase } 1500000 \mathrm{IU} \text { ( } 3 \text { boluses } \\
\quad+25 \text {-min infusion) }\end{array}$ & Systemic \\
\hline $\begin{array}{l}\text { Goldhaber } \\
\text { et }\left.^{4}\right|^{46}\end{array}$ & $\begin{array}{l}\text { Thrombolytic plus UFH }(n=36) \\
\text { thrombolytic alone }(n=17) \\
\text { UFH alone }(n=12)\end{array}$ & 50 & 29.3 & $\begin{array}{l}\text { Proximal DVT, defined as } \\
\text { thromboses of the popliteal or } \\
\text { more proximal veins with or } \\
\text { without concomitant calf vein } \\
\text { thrombosis }\end{array}$ & $\mathrm{rt}-\mathrm{PA} 1.2 \mathrm{mg} / \mathrm{kg}$ (24-h infusion) & Systemic \\
\hline Kakkar et $\mathrm{al}^{47}$ & $\begin{array}{l}\text { Thrombolytic plus UFH }(n=10) \text {; } \\
\text { UFH alone }(n=10)\end{array}$ & 54 & 60 & Any DVT (lower limb) & $\begin{array}{l}\text { Streptokinase, dose not well } \\
\text { defined (at least } 140000 \mathrm{IU} \text { ) } \\
\quad \text { (bolus }+ \text { infusions every } 6 \mathrm{~h} \text { ) }\end{array}$ & Systemic \\
\hline Kiil et $\mathrm{al}^{48}$ & $\begin{array}{l}\text { Thrombolytic plus UFH }(n=11) \text {; } \\
\text { UFH alone }(n=8)(9 \text { patients } \\
\text { were originally allocated but } 1 \\
\text { was excluded after } \\
\text { randomization) }\end{array}$ & 66.5 & 30 & Any DVT (lower limb) & $\begin{array}{l}\text { Urokinase } 200000 \text { IU (24-h } \\
\text { infusion) }\end{array}$ & Systemic \\
\hline Marder et $\mathrm{al}^{49}$ & $\begin{array}{l}\text { Thrombolytic plus UFH }(\mathrm{n}=15) \\
\quad(12 \text { randomized and } 3 \text { added } \\
\text { after randomization); UFH alone } \\
(\mathrm{n}=12)\end{array}$ & 52.4 & 30 & Any DVT (upper or lower limb) & $\begin{array}{l}\text { Streptokinase } 7450000 \mathrm{IU} \text { (bolus } \\
\quad+72 \text {-h infusion) }\end{array}$ & Systemic \\
\hline Schulman et $\mathrm{al}^{50}$ & $\begin{array}{l}\text { Thrombolytic plus UFH }(n=17) \text {; } \\
\text { heparin alone }(n=19)\end{array}$ & 55.5 & 50.3 & $\begin{array}{l}\text { Distal DVT, defined as verified } \\
\text { DVT of the calf, not extending } \\
\text { above the knee joint }\end{array}$ & $\begin{array}{l}\text { Streptokinase } 1250000 \text { to } \\
8450000 \text { IU (bolus }+12-\mathrm{h} \\
\text { infusion for } 1 \text { to } 7 \mathrm{~d} \text { ) }\end{array}$ & Systemic \\
\hline $\begin{array}{l}\text { Schweizer } \\
\text { et al }\end{array}$ & $\begin{array}{l}\text { Thrombolytic plus UFH }(n=46) \text {; } \\
\text { UFH alone }(n=23)\end{array}$ & 40 & 69.5 & $\begin{array}{l}\text { Proximal DVT, defined as lower } \\
\text { leg and popliteal thromboses }\end{array}$ & $\begin{array}{l}\text { rt-PA }(n=23) 140 \mathrm{mg}(4-\mathrm{h} \\
\text { infusions for } 7 \mathrm{~d}) ; \text { urokinase } \\
(\mathrm{n}=23) 16800000 \mathrm{IU} \\
\text { (7-dinfusion) }\end{array}$ & $\begin{array}{l}\text { Locoregional intervention } \\
\text { infused in the dorsal pedal } \\
\text { vein of the affected limb }\end{array}$ \\
\hline $\begin{array}{l}\text { Schweizer } \\
\text { et } \mathrm{al}^{52}\end{array}$ & $\begin{array}{l}\text { Thrombolytic plus UFH }(n=200) \\
\text { UFH alone }(n=50)\end{array}$ & 39.6 & 28.4 & $\begin{array}{l}\text { Proximal DVT, defined as } \\
\text { thrombosis of the popliteal or } \\
\text { more proximal veins with or } \\
\text { without concomitant calf vein } \\
\text { thrombosis }\end{array}$ & $\begin{array}{l}\text { rt-PA }(n=50) 80-140 \mathrm{mg} \text { (4-h } \\
\text { infusions for } 4 \text { to } 7 \mathrm{~d}) ; \text { urokinase } \\
(\mathrm{n}=100) 16800000 \mathrm{IU} \\
(7-\mathrm{d} \text { infusion); streptokinase } \\
(\mathrm{n}=50) 21000000 \mathrm{IU} \\
(6-\mathrm{h} \text { infusion for } 7 \mathrm{~d})\end{array}$ & $\begin{array}{l}\text { Systemic and locoregional } \\
\text { intervention infused in the } \\
\text { dorsal pedal vein of the } \\
\text { affected limb }\end{array}$ \\
\hline Tsapogas et $\mathrm{al}^{53}$ & $\begin{array}{l}\text { Thrombolytic plus UFH }(n=19) \text {; } \\
\text { UFH alone }(n=15)\end{array}$ & 57 & 14.7 & Any DVT (lower limb) & $\begin{array}{l}\text { Streptokinase } 7700000 \mathrm{IU} \text { (bolus } \\
\quad+72 \text {-h infusion) }\end{array}$ & Systemic \\
\hline Turpie et $\mathrm{al}^{54}$ & $\begin{array}{l}\text { Thrombolytic plus UFH }(n=41) \\
\text { placebo plus UFH }(n=42)\end{array}$ & NR & NR & $\begin{array}{l}\text { Proximal DVT (lower limb), not } \\
\text { defined }\end{array}$ & $\begin{array}{l}\text { rt-PA } 0.5 \mathrm{mg} / \mathrm{kg} \text { (4- to 8-h infusion } \\
\text { for } 2 \mathrm{~d} \text { ) }\end{array}$ & Systemic \\
\hline Ugurlu et $\mathrm{al}^{55}$ & $\begin{array}{l}\text { Thrombolytic plus UFH }(n=50) \text {; } \\
\text { UFH alone }(n=47)\end{array}$ & NR & NR & $\begin{array}{l}\text { Any DVT (lower limb), not } \\
\text { defined }\end{array}$ & $\begin{array}{l}\text { Streptokinase } 1500000 \text { to } \\
3000000 \text { IU (15- to } 30-\mathrm{h} \\
\text { infusion) }\end{array}$ & Systemic \\
\hline 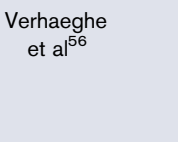 & $\begin{array}{l}\text { Thrombolytic plus UFH }(n=25) \text {; } \\
\text { (14 randomized and } 11 \\
\text { nonrandomized); placebo plus } \\
\text { UFH }(n=7)\end{array}$ & 50 & 42.8 & $\begin{array}{l}\text { Proximal DVT, defined as } \\
\text { popliteal or more proximal } \\
\text { veins of the lower extremities } \\
\text { and/or pelvic veins with or } \\
\text { without calf vein thrombosis }\end{array}$ & $\begin{array}{l}\text { rt-PA } 100 \text { to } 150 \mathrm{mg} \text { ( } 2 \text { boluses on } \\
\text { consecutive days) }\end{array}$ & Systemic \\
\hline
\end{tabular}

NR, not reported. 
Table 2. (continued)

\begin{tabular}{|c|c|c|c|c|c|c|}
\hline References & No. of participants & $\begin{array}{l}\text { Mean } \\
\text { age, y }\end{array}$ & $\begin{array}{l}\text { Females in } \\
\text { study, } \%\end{array}$ & DVT extension & Thrombolytic scheme & Infusion type \\
\hline 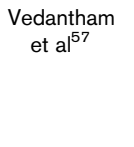 & $\begin{array}{l}\text { Thrombolytic plus LMWH or UFH } \\
\quad(n=336) ; \text { placebo plus LMWH } \\
\text { or UFH }(n=355)\end{array}$ & 52.5 & 38.5 & $\begin{array}{l}\text { Proximal DVT, defined as } \\
\text { thromboses of the femoral, } \\
\text { common femoral, or iliac vein } \\
\text { (with or without other involved } \\
\text { ipsilateral veins) }\end{array}$ & $\begin{array}{l}\text { rt-PA }<35 \mathrm{mg} \text { (through AngioJet, } \\
\text { Trellis, or multi-sidehole catheter } \\
\text { with } 24-\text { to } 30 \text {-h infusion } \\
\text { allowed) }\end{array}$ & Directed \\
\hline Su et $a^{58}$ & $\begin{array}{l}\text { Thrombolytic plus LMWH }(n=73) \text {; } \\
\text { LMWH alone }(n=66)\end{array}$ & 47.7 & 52.5 & $\begin{array}{l}\text { Proximal DVT (lower limb), not } \\
\text { defined }\end{array}$ & $\begin{array}{l}\text { Urokinase } 800000 \text { to } 1200000 \\
\mathrm{IU/d}\end{array}$ & Directed \\
\hline
\end{tabular}

NR, not reported.

performed for long-term outcomes (long-term mortality, PE, recurrent DVT, PTS, and leg ulceration). No significant differences were observed for any of the outcomes compared with the primary analysis. Hence, we assumed low risk of bias because of incomplete outcome data.

\section{Efficacy of thrombolytics for PE}

Mortality. Mortality was reported in 23 of the included trials with a follow-up range of 7 to 90 days (Table 3; Figure 2). Overall, 57 (4.4\%) of the 1295 patients assigned to the control group died compared with $30(2.3 \%)$ of 1297 patients assigned to thrombolytic treatment (RR, 0.61; $95 \% \mathrm{Cl}, 0.4-0.94)$. Certainty of the evidence was judged moderate because of imprecision since OIS was not met (overall, 87 of 2592 included patients died; estimated OIS was 10104 patients).

Nonfatal PE recurrence. PE recurrence was reported in 17 of the included trials with a follow-up range of 7 to 90 days (Table 3; supplemental Figure 3). Overall $48(4.0 \%)$ of the 1178 patients assigned to the control group had PE recurrence compared with 23 $(1.9 \%)$ of 1176 patients assigned to thrombolytic treatment (RR, 0.56; $95 \% \mathrm{Cl}, 0.35-0.89)$. Certainty of the evidence was judged low because of risk of bias and imprecision because most of the data came from unblinded studies, and OIS was not met (overall, 68 of 2354 included patients had recurrent PE; estimated OIS was 11322).

DVT. DVT was reported by 2 of the included trials with a follow-up range of 30 to 90 days (Table 3; supplemental Figure 4). Overall, 2 (3.6\%) of the 55 patients assigned to the control group had DVT compared with 2 (3.5\%) of 57 patients assigned to thrombolytic treatment (RR, $0.92 ; 95 \% \mathrm{Cl}, 0.14-6.03$ ). Certainty of the evidence was judged low because of imprecision since $95 \% \mathrm{Cls}$ included both a significant DVT reduction and increase.

Limited functional class (NYHA III-IV). Limited functional class was reported by 2 of the included trials with a follow-up range of 3 to 24 months (Table 3; supplemental Figure 5). Overall, 28 (7.2\%) of the 398 patients assigned to the control group had limited functional class compared with $23(5.8 \%)$ of 396 patients assigned to thrombolytic treatment (RR, $0.62 ; 95 \% \mathrm{Cl}, 0.17-2.25)$. Certainty of the evidence was judged low because of inconsistency $\left(P^{2}=64 \%\right)$ and imprecision because the $95 \%$ Cls included both significant benefits in terms of preserving a good functional class and absence of benefits.

Subgroup analysis and reporting bias. No significant differences were observed for any of the outcomes when the effects of rtPA, streptokinase, tenecteplase, and urokinase were compared (supplemental Figure 6). Most of the included studies recruited patients with submassive- or intermediate-risk PE or nondefined PE.
One small study included only patients with hemodynamic compromise, and 1 study reported on the subgroup of patients with stable (non-submassive or intermediate risk) PE. There were no significant differences between these subgroups for any of the outcomes (supplemental Figure 7). Thrombolytics were systemically infused in all but 1 of the included studies in which ultrasound-guided directed thrombolysis administration was used. ${ }^{22}$ The estimates of effects reported by that trial did not significantly differ from those of the pooled estimate. Funnel plots did not suggest the presence of publication bias for mortality or PE recurrence outcomes.

\section{Efficacy of thrombolytics for DVT}

Short-term mortality. VTE-related mortality was reported in 12 of the included trials with a median follow-up of 10 days (Table 4; supplemental Figure 8). Overall, 6 (0.8\%) of 708 patients assigned to the control group died compared with $4(0.5 \%)$ of 839 patients assigned to thrombolytic treatment (RR, 0.77; 95\% Cl, 0.26-2.28). Certainty of the evidence was judged low because of risk of bias and imprecision since most of the data came from unblinded studies, and 95\% Cls included both reduction and increase in significant mortality.

Late mortality. Late mortality was reported in 6 of the included trials with a follow-up range of 1 to 6 years (Table 4; supplemental Figure 9). Overall, 22 (4.9\%) of 451 patients assigned to the control group died compared with $19(3.1 \%)$ of 607 patients assigned to thrombolytic treatment (RR, $0.89 ; 95 \% \mathrm{Cl}, 0.47-1.69)$. Certainty of the evidence was judged low because of risk of bias and imprecision.

Nonfatal PE. PE was reported in 9 of the included trials with a follow-up range of 1 week to 6 years (Table 4; supplemental Figure 10). Overall, 15 (3.0\%) of 504 patients assigned to the control group suffered a nonfatal PE compared with 29 (4.4\%) of 661 patients assigned to thrombolytic treatment (RR, 1.33; $95 \% \mathrm{Cl}, 0.71-2.46$ ). Certainty of the evidence was judged low because of risk of bias and imprecision.

Recurrent DVT. Recurrent DVT was reported in 3 of the included trials with a follow-up range of 2 to 6 years (Table 4; supplemental Figure 11). Overall, $40(11.2 \%)$ of 358 patients assigned to the control group suffered DVT recurrence compared with $40(10.9 \%)$ of 368 patients assigned to thrombolytic treatment (RR, $0.99 ; 95 \% \mathrm{Cl}, 0.56-1.76)$. Certainty of the evidence was judged low because of risk of bias and imprecision.

PTS. PTS was reported in 8 of the included trials with a follow-up range of 6 months to 6 years (Table 4; Figure 3). Overall, 359 $(61.8 \%)$ of 581 patients assigned to the control group developed PTS compared with 388 (50.9\%) of 762 patients assigned to 


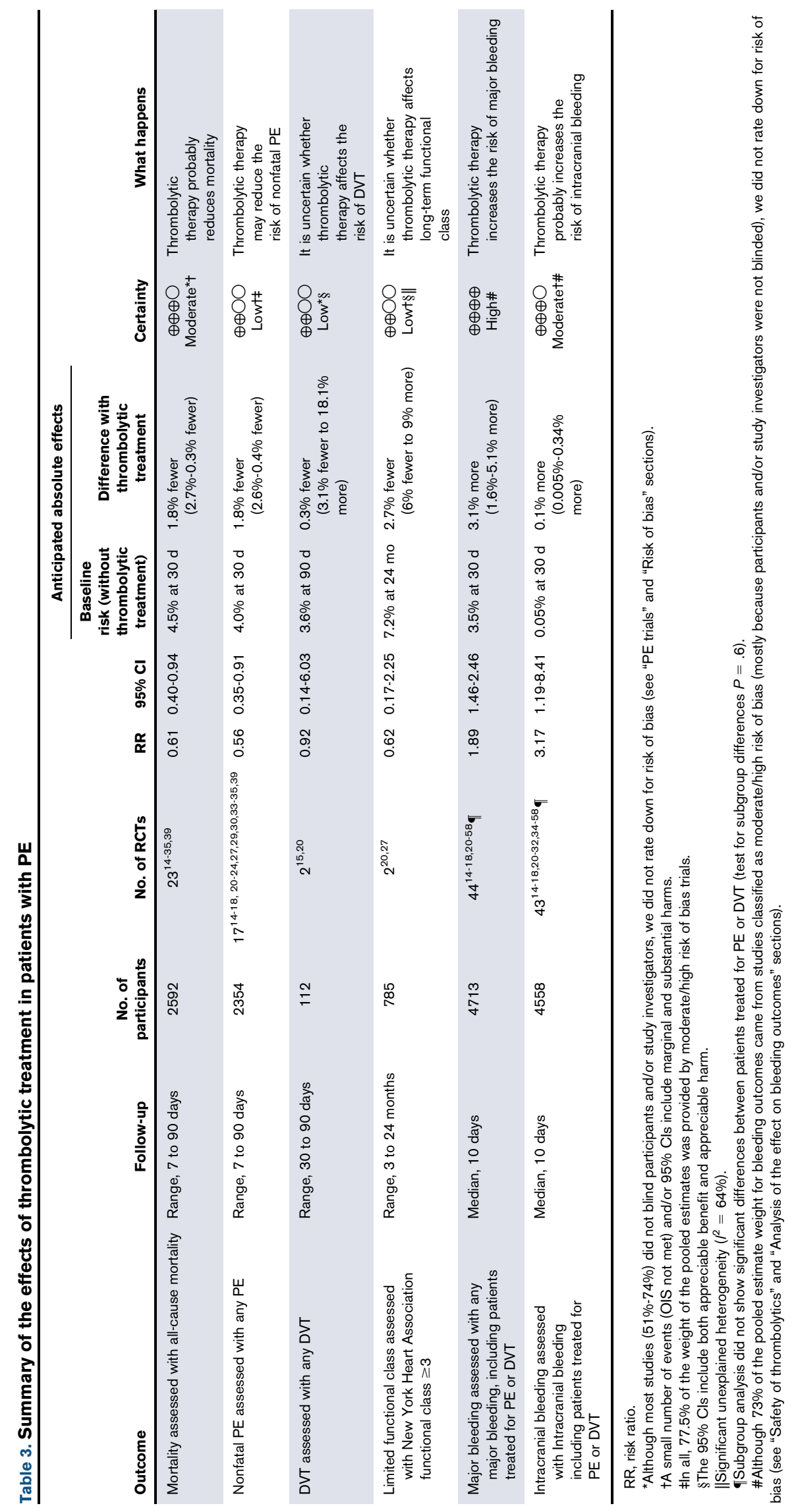




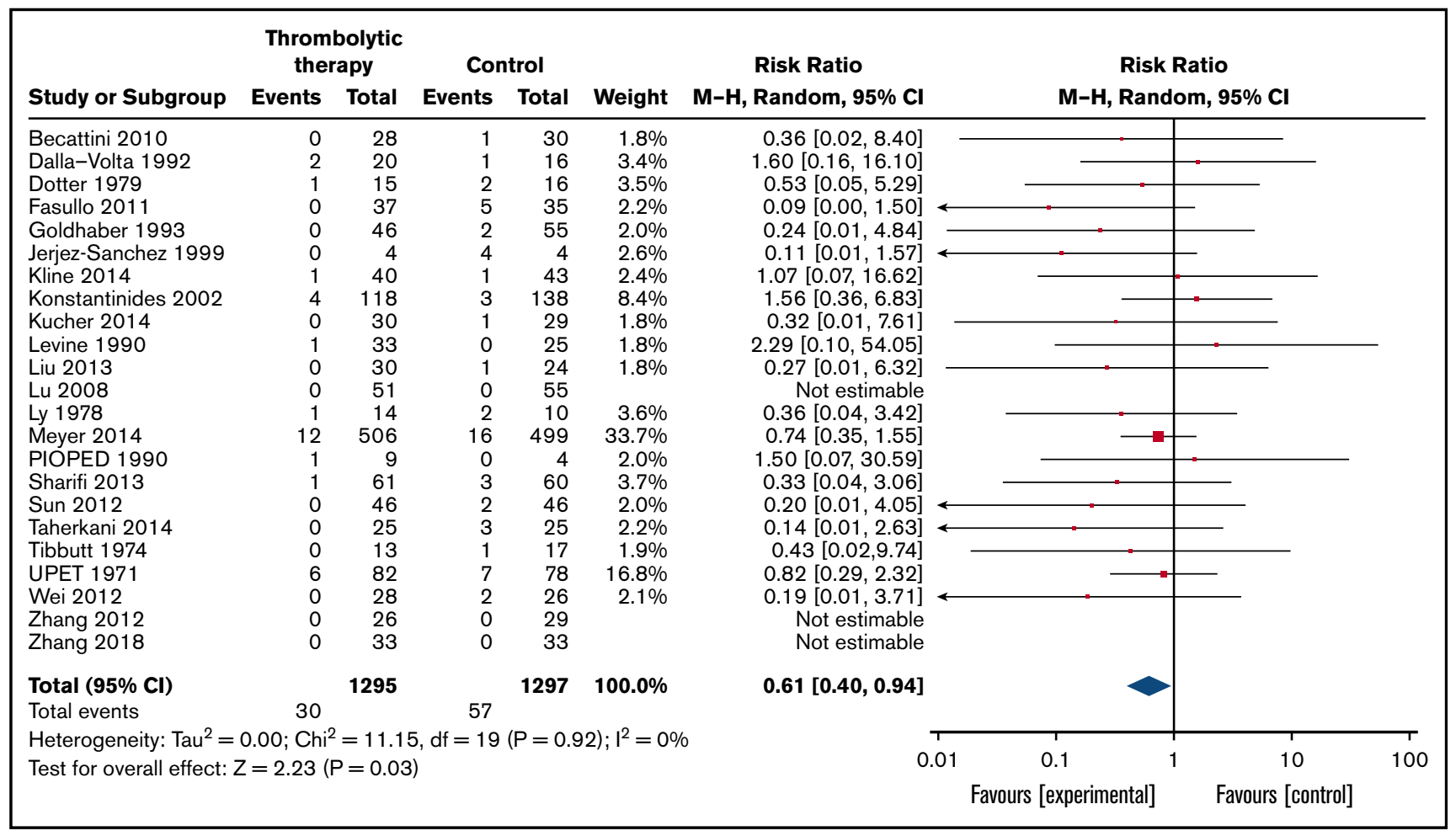

Figure 2. Forest plot of the effects of thrombolytics vs control on mortality in patients with PE. M-H, Mantel-Haenszel.

thrombolytic treatment (RR, 0.70;95\% Cl, 0.59-0.83). Certainty in the estimate of effect was low because of risk of bias and inconsistency $\left(I^{2}=59 \%\right)$ since 1 of the studies (ATTRACT trial) ${ }^{57}$ showed no significant reduction in PTS whereas the rest did.

Leg ulceration. Leg ulceration was reported in 5 of the included trials with a follow-up range of 1 to 6 years (Table 4; supplemental Figure 12). Overall, $22(4.8 \%)$ of the 461 patients assigned to the control group suffered leg ulceration compared with 17 (3.6\%) of 476 patients assigned to thrombolytic treatment $(\mathrm{RR}, 0.75 ; 95 \% \mathrm{Cl}$, 0.39-1.42). Certainty of the evidence was judged low because of risk of bias and imprecision.

Subgroup analysis and reporting bias. Significant heterogeneity was observed in the PTS outcome analysis $\left(I^{2}=57 \%\right)$. Subgroup analysis suggested that inconsistency may be explained by risk of bias because the magnitude of effect was significantly smaller in the only trial in which outcome assessors were blinded ${ }^{57}$ (RR, 0.90; 95\% Cl, 0.78-1.05) as opposed to the pooled estimate in the unblinded trials (RR, $0.64 ; 95 \% \mathrm{Cl}, 0.52-0.79$; test for subgroup differences $P<.01$; supplemental Figure 13). It is noteworthy that this trial was the only one in which a pharmaco-mechanical catheter-directed thrombolytic approach was implemented, which could also explain the observed differences.

No significant differences were observed for any of the outcomes when the effects of rt-PA, streptokinase, and urokinase were compared (supplemental Figure 14). Most of the included studies recruited patients with proximal DVT. One study included only patients with distal DVT, and rest did not specify thrombus location or extension. The comparison between these study subgroups showed no significant differences for any of the outcomes. Seven of the 8 studies that reported on a PTS outcome (96.6\% of the weight of the pooled estimates) included only patients with proximal DVT (supplemental Figure 15).

Thrombolytics were systemically or locally infused in most of the included studies; catheter-directed infusion was used in 4 studies. ${ }^{43,44,57,58}$ No significant differences for any of the outcomes based on infusion strategy were observed (supplemental Figure 16). Funnel plots did not suggest the presence of publication bias for DVT-related mortality or PTS.

\section{Safety of thrombolytics}

Analysis of the effect on bleeding outcomes. No significant differences were observed in the effects of thrombolytics on major bleeding or intracranial bleeding between studies that included patients with DVT or PE (test for interaction $P=.37 ; l^{2}=0 \%$ ); hence, we decided to pool results from all trials reporting bleeding outcome (Tables 1 and 2).

Although 36 of the 44 trials (73\% of the pooled estimate weight) that reported bleeding outcomes were classified as moderate/high risk of bias, we decided not to rate down the certainty of the evidence because no significant differences between trials with moderate/high or low risk of bias were observed in the subgroup analysis for major bleeding or intracranial bleeding (test for interaction $P=.44, I^{2}=0 \%$ and $P=.47, I^{2}=0 \%$, respectively).

Major bleeding. Major bleeding was reported in 44 of the included trials (19 in patients with DVT and 25 in patients with PE) with a median of follow-up of 10 days (Tables 3 and 4; Figure 4). Overall, 78 (3.5\%) of 2246 patients assigned to the control group suffered major bleeding compared with $196(7.9 \%)$ of 2467 


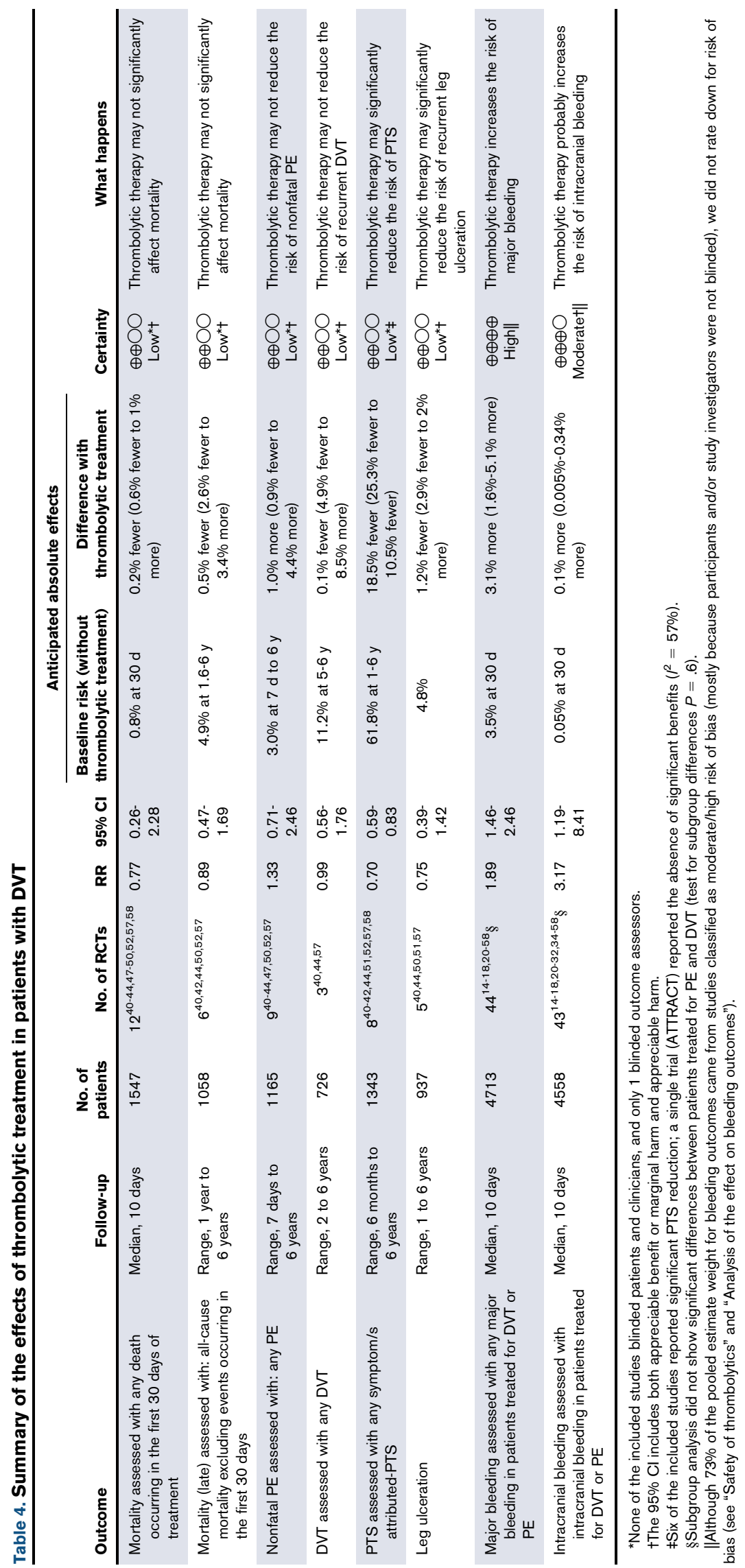




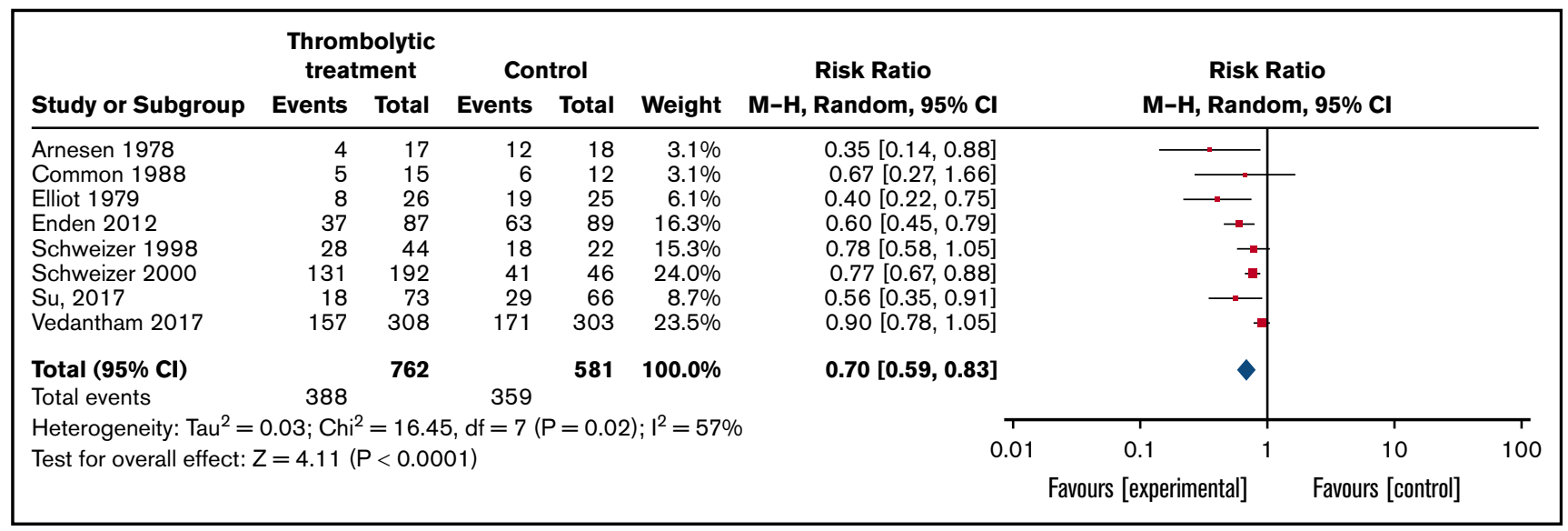

Figure 3. Forest plot of the effects of thrombolytics vs control on PTS in patients with DVT.

patients assigned to thrombolytic treatment $(\mathrm{RR}, 1.89 ; 95 \% \mathrm{Cl}$, 1.46-2.46). Certainty of the evidence was judged high.

Intracranial bleeding. Intracranial bleeding was reported in 43 of the included trials (19 in patients with DVT and 24 in patients with PE) with a median of follow-up of 10 days (Tables 3 and 4; supplemental Figure 17). Overall, $1(0.05 \%)$ of the 2170 patients assigned to the control group suffered intracranial bleeding compared with $18(0.8 \%)$ of 2388 patients assigned to thrombolytic treatment $(\mathrm{RR}, 3.17 ;(95 \% \mathrm{Cl}, 1.19-8.41)$. Certainty of the evidence was judged moderate because of imprecision since absolute effects $95 \% \mathrm{Cls}$ included both very small harms (1 more intracranial bleed for 20000 patients treated) and substantial harms (1 more intracranial bleed for 294 patients treated).

Subgroup analysis and reporting bias. Subgroup analysis comparing different thrombolytic agents suggested that tenecteplase may be associated with an increase in bleeding risk compared with urokinase, rt-PA, or streptokinase (test for interaction $P=.01$; $I^{2}=73.1 \%$ ) (supplemental Figure 18). No significant differences between catheter-directed and systemic infusion strategies were observed for major bleeding or intracranial bleeding (supplemental Figure 19). Funnel plots did not suggest the presence of publication bias.

\section{Discussion}

This systematic review reports moderate certainty evidence that thrombolytic therapy reduces short-term mortality in patients with submassive- or intermediate-risk PE, and low certainty evidence that thrombolytic therapy reduces PTS in patients with proximal DVT at the expense of an increase in major bleeding episodes, including intracranial bleeding.

In PE trials, mortality risk in the control group (baseline risk) varied significantly between studies from $2.3 \%{ }^{20}$ to $100 \% .^{19}$ In patients with a high risk of dying from $\mathrm{PE}$, the reduction of mortality because of the use of thrombolytics may be substantial, whereas in patients with a low risk of dying, it would be much smaller. This distinction of the baseline risk of patients is relevant, because thrombolytics were found to increase the risk of major bleeding. Thus, patients at high risk of dying who have a low risk of bleeding may benefit from thrombolytics, whereas in patients with a low risk of dying and a high risk of bleeding, thrombolytics may result in net harm. In line with this finding, most recently published guidelines on the topic recommend thrombolytic treatment only for patients with high-risk PE (hemodynamic instability). ${ }^{59}$

In patients with DVT, the possible benefits observed in terms of PTS risk reduction would probably be counterbalanced by increased bleeding risk in most clinical situations. Again, the baseline risk distinction is probably important. As in most patients, the risk of serious complication as a result of DVT is small, and the risk of bleeding probably outweighs the potential benefits. However, patients with high baseline risk of developing PTS or limbthreatening DVT and low risk of bleeding may benefit from thrombolytics.

Catheter-directed thrombolysis (CDT) aims to reduce bleeding complications associated with systemic thrombolysis while preserving its beneficial effects. Although its implementation is more demanding on resources than systemic thrombolytic infusion, this technique has gained popularity in the last decade, especially for treating patients with DVT. ${ }^{60-62}$ Even though subgroup analysis did not suggest that CDT infusion strategy was more effective or safer than systemic strategy, these analyses were probably underpowered, and a differential effect cannot be excluded. Our search strategy identified 5 additional randomized controlled trials (not included in the analysis for this review because of active comparators), ${ }^{63-67}$ in which a CDT strategy was compared with a systemic strategy. No significant differences were reported in any of those trials, although they were small and possibly underpowered to detect an effect (26 major bleeding events in total). In addition, we identified 2 propensity score-adjusted, registry-based, observational studies that included 6244 patients with $P E$ that reported no significant differences in terms of major bleeding between patients treated with CDT infusion and systemic thrombolytic infusion. ${ }^{68,69}$

Our systematic review has several strengths. First, it provides the most comprehensive and trustworthy body of evidence to date, including 9 studies $24,25,30,34,35,36,38,57,58$ not included in the most recent reviews. ${ }^{7,8}$ Although the conclusions of our systematic review in terms of the effect of the intervention are not different from the conclusions of previous reviews addressing the same questions, we believe that our review provides a more comprehensive perspective for the body of evidence supported in novel 


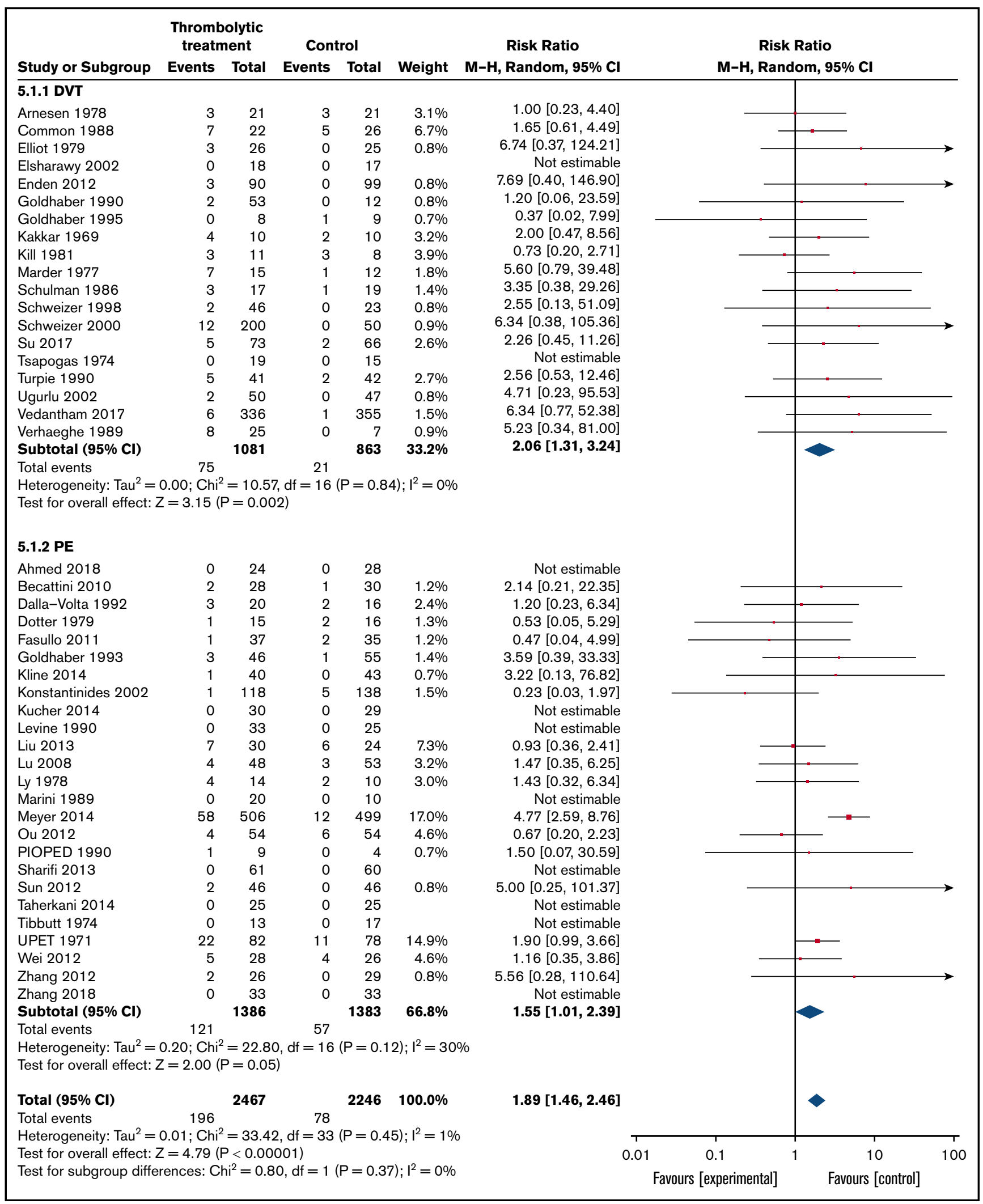

Figure 4. Forest plot of the effects of thrombolytics vs control on major bleeding in patients with VTE by primary event (PE or DVT). 
analytical approaches for 3 reasons: (1) although most of the included studies have methodologic limitations, we weighted the potential impact of those limitations to the pooled estimates and evaluated the certainty of the evidence accordingly; (2) we considered multiple critical outcomes related to the effect of thrombolytic treatment in patients with DVT; and (3) we combined studies that included patients with PE and DVT and ended up with high certainty regarding the effects of thrombolytics on major bleeding outcomes.

Potential limitations are related to the variability in the inclusion criteria and the treatment regimens used in the different primary studies that were included. Although we did not find significant subgroup effects, our approach might not have captured the diversity in treatment schemes and definitions that were used (ie, we did not perform a subgroup analysis considering thrombolytic dose). In addition, although the certainty in the estimates of effects was low for most efficacy outcomes, we did not include observational studies in our review.

In conclusion, thrombolytic therapy probably reduces mortality in patients with submassive- or intermediate-risk PE and may reduce the risk of PTS in patients with proximal DVT while significantly increasing the risk of major bleeding, including intracranial bleeding. The balance between benefits and risks of thrombolytic therapy in the analyzed scenarios is profoundly influenced by the baseline risks of the relevant outcomes. Stakeholders involved in the decisionmaking process would need to weigh these effects to define which clinical scenarios merit use of thrombolytic therapy for patients presenting with acute VTE.

\section{Acknowledgment}

This systematic review was performed as part of the ASH Clinical Practice Guidelines on VTE.

Supported by the American Society of Hematology, which funded the entire guideline development process.

\section{Authorship}

Contribution: A.l. contributed to study design, study selection, collection of data, statistical analyses, data interpretation, and writing the manuscript; J.M.C., F.P., L.L., and J.W. contributed to study selection and data collection; W.A., D.M.W., M.R.J., S.S.,V.M., P.V., Y.Z., and G.R. interpreted the data and provided vital reviews of the manuscript; and R.N., H.J.S., I.N., and W.W. contributed to study design, interpreted the data, and provided vital reviews of the manuscript.

Conflict-of-interest disclosure: The authors declare no competing financial interests.

ORCID profiles: A.I., 0000-0001-9053-4396; J.M.C., 00000003-2041-1803; D.M.W., 0000-0002-3930-8358; M.R.J., 00000002-3772-5887; V.M., 0000-0003-0410-8089; W.W., 00000001-6576-1650; H.J.S., 0000-0003-3211-8479.

Correspondence: Ariel Izcovich, Internal Medicine Service, German Hospital, Pueyrredón 1640, C1118 AAT Buenos Aires, Argentina; e-mail: ariel.izcovich@gmail.com.

\section{References}

1. Raskob GE, Angchaisuksiri P, Blanco AN, et al; ISTH Steering Committee for World Thrombosis Day. Thrombosis: a major contributor to global disease burden. Arterioscler Thromb Vasc Biol. 2014;34(11):2363-2371.

2. Khan F, Rahman A, Carrier M, et al; MARVELOUS Collaborators. Long term risk of symptomatic recurrent venous thromboembolism after discontinuation of anticoagulant treatment for first unprovoked venous thromboembolism event: systematic review and meta-analysis. BMJ. 2019; $366: 14363$.

3. Kahn SR, Shrier I, Julian JA, et al. Determinants and time course of the postthrombotic syndrome after acute deep venous thrombosis. Ann Intern Med. 2008;149(10):698-707.

4. Schulman S, Lindmarker P, Holmström M, et al. Post-thrombotic syndrome, recurrence, and death 10 years after the first episode of venous thromboembolism treated with warfarin for 6 weeks or 6 months. J Thromb Haemost. 2006;4(4):734-742.

5. Higgins JPT, Green S, eds, Cochrane Handbook for Systematic Reviews of Interventions Version 5.1.0 [updated March 2011]. The Cochrane Collaboration, 2011. http://handbook-5-1.cochrane.org/. Accessed 18 February 2019.

6. Moher D, Liberati A, Tetzlaff J, Altman DG; PRISMA Group. Preferred reporting items for systematic reviews and meta-analyses: the PRISMA statement. PLoS Med. 2009;6(7):e1000097.

7. Hao Q, Dong BR, Yue J, Wu T, Liu GJ. Thrombolytic therapy for pulmonary embolism. Cochrane Database Syst Rev. 2015;9(9):CD004437.

8. Watson L, Broderick C, Armon MP. Thrombolysis for acute deep vein thrombosis. Cochrane Database Syst Rev. 2016;11:CD002783.

9. AkI EA, Sun X, Busse JW, et al. Specific instructions for estimating unclearly reported blinding status in randomized trials were reliable and valid. $J$ Clin Epidemiol. 2012;65(3):262-267.

10. Guyatt $\mathrm{GH}$, Ebrahim S, Alonso-Coello $\mathrm{P}$, et al. GRADE guidelines 17: assessing the risk of bias associated with missing participant outcome data in a body of evidence. J Clin Epidemiol. 2017;87:14-22.

11. Guyatt GH, Oxman AD, Kunz R, et al. GRADE guidelines 6. Rating the quality of evidence-imprecision. J Clin Epidemiol. 2011;64(12):1283-1293.

12. Guyatt GH, Oxman AD, Montori V, et al. GRADE guidelines: 5. Rating the quality of evidence-publication bias. J Clin Epidemiol. 2011;64(12): 1277-1282.

13. Guyatt GH, Oxman AD, Santesso N, et al. GRADE guidelines: 12. Preparing summary of findings tables-binary outcomes. J Clin Epidemiol. 2013;66(2):158-172.

14. Becattini C, Agnelli G, Salvi A, et al; TIPES Study Group. Bolus tenecteplase for right ventricle dysfunction in hemodynamically stable patients with pulmonary embolism. Thromb Res. 2010;125(3):e82-e86. 
15. Dalla-Volta S, Palla A, Santolicandro A, et al. PAIMS 2: alteplase combined with heparin versus heparin in the treatment of acute pulmonary embolism. Plasminogen Activator Italian Multicenter Study 2. J Am Coll Cardiol. 1992;20(3):520-526.

16. Dotter CT, Seaman AJ, Rösch J, Porter JM. Streptokinase and heparin in the treatment of pulmonary embolism: A randomized comparison. Vasc Endovasc Surg. 1979;13(1):42-52.

17. Fasullo S, Scalzo S, Maringhini G, et al. Six-month echocardiographic study in patients with submassive pulmonary embolism and right ventricle dysfunction: Comparison of thrombolysis with heparin. Am J Med Sci. 2011;341(1):33-39.

18. Goldhaber SZ, Haire WD, Feldstein ML, et al. Alteplase versus heparin in acute pulmonary embolism: randomised trial assessing right-ventricular function and pulmonary perfusion. Lancet. 1993;341(8844):507-511.

19. Jerjes-Sanchez C, Ramírez-Rivera A, de Lourdes García M, et al. Streptokinase and heparin versus heparin alone in massive pulmonary embolism: A randomized controlled trial. J Thromb Thrombolysis. 1995;2(3):227-229.

20. Kline JA, Nordenholz KE, Courtney DM, et al. Treatment of submassive pulmonary embolism with tenecteplase or placebo: Cardiopulmonary outcomes at 3 months: Multicenter double-blind, placebo-controlled randomized trial. J Thromb Haemost. 2014;12(4):459-468.

21. Konstantinides S, Geibel A, Heusel G, Heinrich F, Kasper W. Heparin plus alteplase compared with heparin alone in patients with submassive pulmonary embolism. N Engl J Med. 2002;347(15):1143-1150.

22. Kucher $\mathrm{N}$, Boekstegers $\mathrm{P}$, Müller OJ, et al. Randomized, controlled trial of ultrasound-assisted catheter-directed thrombolysis for acute intermediate-risk pulmonary embolism. Circulation. 2014;129(4):479-486.

23. Levine M, Hirsh J, Weitz J, et al. A randomized trial of a single bolus dosage regimen of recombinant tissue plasminogen activator in patients with acute pulmonary embolism. Chest. 1990;98(6):1473-1479.

24. Liu LH, Lu SJ, Liu Z. A study of thrombolysis and anticoagulation in patients with sub large area pulmonary embolism [in Chinese]. Chongqing Med J. 2013;42(11):1288-1290.

25. Lu WH, Tang ZZ, Ma Y, Yu ZH. Long term prognosis of thrombolytic therapy with urokinase and/or anticoagulation submassive pulmonary embolism [in Chinese]. Clin J Med Officer. 2008;36(3):340-342.

26. Ly B, Arnesen H, Eie H, Hol R. A prospective study of streptokinase and heparin in the treatment of major pulmonary embolism. Acta Med Scand. 1978; 203:465-470.

27. Meyer G, Vicaut E, Danays T, et al; PEITHO Investigators. Fibrinolysis for patients with intermediate-risk pulmonary embolism. N Engl J Med. 2014; 370(15):1402-1411.

28. Tissue plasminogen activator for the treatment of acute pulmonary embolism. A collaborative study by the PIOPED Investigators. Chest. 1990;97(3): 528-533.

29. Sharifi M, Bay C, Skrocki L, Rahimi F, Mehdipour M; "MOPETT" Investigators. Moderate pulmonary embolism treated with thrombolysis (from the "MOPETT" Trial). Am J Cardiol. 2013;111(2):273-277.

30. Sun RQ. Urokinase combined with low molecular weight heparin and warfarin in the treatment of sub large area pulmonary embolism [in Chinese]. Strait Pharm J. 2012;24(5):130-131.

31. Taherkhani M, Taherkhani A, Hashemi SR, et al. Thrombolytic-plus-anticoagulant therapy versus anticoagulant-alone therapy in submassive pulmonary thromboembolism (TVASPE Study): A randomized clinical trial. J Tehran Heart Cent. 2014;9(3):104-108.

32. Tibbutt DA, Davies JA, Anderson JA, et al. Comparison by controlled clinical trial of streptokinase and heparin in treatment of life-threatening pulmonay embolism. BMJ. 1974;1(5904):343-347.

33. Urokinase pulmonary embolism trial. Phase 1 results: a cooperative study. JAMA. 1970;214(12):2163-2172.

34. Wei L, Sun F. Curative effect of thrombolysis and anticoagulant therapy on submassive/intermediate risk pulmonary embolism patients [in Chinese]. Int $J$ Resp. 2012;32(19):1471-1473.

35. Zhang X, Jiang S, Jiang L, Shan F, Li Z, Qin M. Low dose RT PA thrombolysis for acute sub large area pulmonary embolism: a study of 26 elderly patients [in Chinese]. Shandong Yiyao. 2012;52(3):69-70.

36. Ou Y. Analysis of therapeutic effect of urokinase combined with sequential anti-coagulation in sub-massive pulmonary embolism [in Chinese]. $J$ Hainan Med Coll. 2012;18(7):897-898.

37. Marini C, Di Ricco G, Rossi G, Rindi M, Palla R, Giuntini C. Fibrinolytic effects of urokinase and heparin in acute pulmonary embolism: a randomized clinical trial. Respiration. 1989;54(3):162-173.

38. Ahmed MA, Abdelsalam SI, Elmorsy RA. Value of thrombolytic therapy for submassive pulmonary embolism patients. Egypt J Chest Dis Tuberc. 2018; 67(4):413-418.

39. Zhang LY, Gao BA, Jin Z, et al. Clinical efficacy of low dose recombinant tissue-type plasminogen activator for the treatment of acute intermediate-risk pulmonary embolism. Saudi Med J. 2018;39(11):1090-1095.

40. Arnesen H, Heilo A, Jakobsen E, Ly B, Skaga E. A prospective study of streptokinase and heparin in the treatment of deep vein thrombosis. Acta Med Scand. 1978;203(6):457-463.

41. Common HH, Seaman AJ, Rösch J, Porter JM, Dotter CT. Deep vein thrombosis treated with streptokinase or heparin. Follow-up of a randomized study. Angiology. 1976;27(11):645-654.

42. Elliot MS, Immelman EJ, Jeffery $\mathrm{P}$, et al. A comparative randomized trial of heparin versus streptokinase in the treatment of acute proximal venous thrombosis: an interim report of a prospective trial. Br J Surg. 1979;66(12):838-843. 
43. Elsharawy M, Elzayat E. Early results of thrombolysis vs anticoagulation in iliofemoral venous thrombosis. A randomised clinical trial. Eur J Vasc Endovasc Surg. 2002;24(3):209-214.

44. Enden T, Haig Y, Kløw NE, et al; CaVenT Study Group. Long-term outcome after additional catheter-directed thrombolysis versus standard treatment for acute iliofemoral deep vein thrombosis (the CaVenT study): a randomised controlled trial. Lancet. 2012;379(9810):31-38.

45. Goldhaber SZ, Hirsch DR, MacDougall RC, Polak JF, Creager MA. Bolus recombinant urokinase versus heparin in deep venous thrombosis: a randomized controlled trial. Am Heart J. 1996;132(2 pt 1):314-318.

46. Goldhaber SZ, Meyerovitz MF, Green D, et al. Randomized controlled trial of tissue plasminogen activator in proximal deep venous thrombosis. Am J Med. 1990;88(3):235-240.

47. Kakkar VV, Flanc C, Howe CT, O'Shea M, Flute PT. Treatment of deep vein thrombosis. A trial of heparin, streptokinase, and arvin. BMJ. 1969;1(5647): 806-810.

48. Kiil J, Carvalho A, Saksø P, Nielsen HO. Urokinase or heparin in the management of patients with deep vein thrombosis? Acta Chir Scand. 1981;147(7): 529-532.

49. Marder VJ, Soulen RL, Atichartakarn V, et al. Quantitative venographic assessment of deep vein thrombosis in the evaluation of streptokinase and heparin therapy. J Lab Clin Med. 1977;89(5):1018-1029.

50. Schulman S, Granqvist S, Juhlin-Dannfelt A, Lockner D. Long-term sequelae of calf vein thrombosis treated with heparin or low-dose streptokinase. Acta Med Scand. 1986;219(4):349-357.

51. Schweizer J, Elix H, Altmann E, Hellner G, Forkmann L. Comparative results of thrombolysis treatment with rt-PA and urokinase: a pilot study. Vasa. 1998; 27(3):167-171.

52. Schweizer J, Kirch W, Koch R, et al. Short- and long-term results after thrombolytic treatment of deep venous thrombosis. J Am Col/ Cardiol. 2000;36(4): 1336-1343.

53. Tsapogas MJ, Peabody RA, Wu KT, Karmody AM, Devaraj KT, Eckert C. Controlled study of thrombolytic therapy in deep vein thrombosis. Surgery. 1973; 74(6):973-984.

54. Turpie AG, Levine MN, Hirsh J, et al. Tissue plasminogen activator (rt-PA) vs heparin in deep vein thrombosis. Results of a randomized trial. Chest. 1990; 97(4 suppl):172S-175S.

55. Ugurlu B, Kazaz H, Oto O, Hazan E, Sariosmanoğlu N. Low dose systemic thrombolytic therapy for treatment of deep venous thrombosis. J Cardiovasc Surg (Torino). 2002;43(6):881-885

56. Verhaeghe R, Besse $\mathrm{P}$, Bounameaux $\mathrm{H}$, Marbet GA. Multicenter pilot study of the efficacy and safety of systemic rt-PA administration in the treatment of deep vein thrombosis of the lower extremities and/or pelvis. Thromb Res. 1989;55(1):5-11.

57. Vedantham S, Goldhaber SZ, Julian JA, et al; ATTRACT Trial Investigators. Pharmacomechanical catheter-directed thrombolysis for deep-vein thrombosis. N Engl J Med. 2017;377(23):2240-2252.

58. Su SF, Tian YF, Chen LB, Yan B. [Comparison of therapeutic efficacy of anticoagulation and its combination with catheter-directed thrombolysis for deep venous thrombosis of lower extremities] [in Chinese]. Zhongguo Shi Yan Xue Ye Xue Za Zhi. 2017;25(5):1509-1513.

59. Konstantinides SV, Meyer G, Becattini C, et al; ESC Scientific Document Group. 2019 ESC Guidelines for the diagnosis and management of acute pulmonary embolism developed in collaboration with the European Respiratory Society (ERS). Eur Heart J. 2020;41(4):543-603.

60. Bashir R, Zack CJ, Zhao H, Comerota AJ, Bove AA. Comparative outcomes of catheter-directed thrombolysis plus anticoagulation vs anticoagulation alone-treat lower-extremity proximal deep vein thrombosis. JAMA Intern Med. 2014;174(9):1494-1501.

61. Adusumalli S, Geller BJ, Yang L, Giri J, Groeneveld PW. Trends in catheter-directed thrombolysis and systemic thrombolysis for the treatment of pulmonary embolism. Am Heart J. 2019;207:83-85.

62. Gayou EL, Makary MS, Hughes DR, et al. Nationwide trends in use of catheter-directed therapy for treatment of pulmonary embolism in Medicare beneficiaries from 2004-2016. J Vasc Interv Radiol. 2019;30(6):801-806.

63. Macovei L, Presura RM, Arsenescu Georgescu C. Systemic or local thrombolysis in high-risk pulmonary embolism. Cardiol J. 2015;22(4):467-474.

64. Gong Y, Gu Y, Chen Z, Yu Z, Lv J. Thrombolysis in the treatment of lower extremity deep vein thrombosis [in Chinese]. J Chinese Physician. 2005;7(8): 1101.

65. Yu X, Zhou C, Chen X. A clinical study on the treatment of deep vein thrombosis of lower extremity by interventional urokinase thrombolysis via popliteal vein [in Chinese]. J South Med Univ. 2009;(9):1930-1931.

66. Zhou ZX, Pan CQ, Fu FY, Lin ZQ, Liu ZJ. [Efficacy of regional administration of urokinase and argatroban via small saphenous vein catheter for treatment of acute deep venous thrombosis in the lower limb] [in Chinese]. Nan Fang Yi Ke Da Xue Xue Bao. 2011;31(3):539-543.

67. Luo QC, Zeng QH. A study of inferior vena cava filter combined with catheter directed thrombolysis in patients with deep vein thrombosis of lower extremity [in Chinese]. Jiangxi Med J. 2011;46(4):303-306.

68. Arora S, Lahewala S, Patel P, et al. Catheter-directed thrombolysis versus systemic thrombolysis in pulmonary embolism: Predictors of in-hospital mortality and major bleeding [abstract]. Circulation. 2016;134(suppl 1). Abstract A15240.

69. Geller BJ, Adusumalli S, Yang L, Groeneveld PW, Giri J. Catheter directed thrombolysis versus systemic thrombolysis for the treatment of pulmonary embolism [abstract]. Circulation. 2017;136(suppl 1). Abstract 18461. 